\title{
Les concepts dans la recherche en didactique du français
}

Émergence et création d'un champ épistémique

Concepts in research in didactics of french. Emergence and creation of an epistemic field

\section{Marie-Cécile Guernier}

\section{(2) OpenEdition}

Journals

Édition électronique

URL : https://journals.openedition.org/pratiques/9443

DOI : 10.4000 /pratiques. 9443

ISSN : 2425-2042

Éditeur

Centre de recherche sur les médiations (CREM)

Référence électronique

Marie-Cécile Guernier, «Les concepts dans la recherche en didactique du français », Pratiques [En ligne], 189-190 | 2021, mis en ligne le 09 juillet 2021, consulté le 23 juillet 2021. URL : http:// journals.openedition.org/pratiques/9443; DOI : https://doi.org/10.4000/pratiques.9443

Ce document a été généré automatiquement le 23 juillet 2021.

(c) Tous droits réservés 


\title{
Les concepts dans la recherche en didactique du français
}

\author{
Émergence et création d'un champ épistémique \\ Concepts in research in didactics of french. Emergence and creation of an \\ epistemic field
}

Marie-Cécile Guernier

1 Les textes fondateurs qui ont accompagné la constitution de la didactique du français comme champ de recherche insistent sur la nécessité que celle-ci engage un travail épistémologique d'élaboration de concepts pour se constituer "scientifiquement " (Chiss, David \& Reuter, 2008 [1995]; Marquillo Larruy, 2001). Cette nécessité est régulièrement réitérée (Reuter, 2013 [2007]; Dumortier, 2008; Daunay, Reuter \& Schneuwly, 2011; Daunay \& Grossmann, 2012a) ainsi que l'affirmation que cette élaboration conceptuelle est une problématique majeure, d'une part parce qu'elle est par définition le mouvement même de la recherche, d'autre part parce qu'elle est le lieu de débats sur ses enjeux. Dans le cadre de la réflexion sur l'élaboration épistémologique de la didactique du français proposée par l'AIRDF pour son $14^{\mathrm{e}}$ congrès en août 2019 à Lyon, nous avons souhaité tenter une synthèse dont cet article rend compte. La question y est abordée selon trois aspects : l'aspect diachronique, en examinant, à partir de publications qui ont compté dans le développement de ce champ épistémique, comment la question a été discutée au fur et à mesure de la constitution de ce champ, l'aspect épistémologique en examinant d'une part comment le concept de concept y est appréhendé (aspect $n^{\circ} 2$ ), d'autre part quelle heuristique développe l'activité de conceptualisation (aspect $\mathrm{n}^{\circ} 3$ ). Bien évidemment, l'ampleur de la question empêche l'exhaustivité, et nous nous en tiendrons aux discussions tenues dans le cadre $\mathrm{du}$ congrès de l'AIRDF. Cet article est complémentaire de celui paru dans le $\mathrm{n}^{\circ} 67$ de $\mathrm{L} a$ Lettre de l'AIRDF dans lequel nous analysons (Guernier, 2020) les propositions de communications au colloque d'août 2019. 


\section{Construction épistémologique : débats et enjeux. Quelques repères diachroniques}

2 Les textes fondateurs qui ont accompagné la constitution de la didactique du français comme champ de recherche indiquent, bien évidemment, que pour se constituer « scientifiquement » la didactique du français doit engager un travail épistémologique d'élaboration de concepts. Ce principe et cette nécessité font consensus. Pour autant la question fait débat, et quelquefois dissensus, au sein de la communauté des chercheurs en didactique du français. Sont en question, en particulier, les enjeux de la constitution de la didactique du français sur le modèle d'une discipline universitaire de recherche, et est ainsi interrogée la nature même de la recherche en didactique du français. Pour rendre compte de ces discussions, nous avons retenu dans cette partie des textes (articles ou chapitres d'ouvrage) qui débattent explicitement de la question de l'élaboration épistémologique de la didactique du français.

\subsection{5 - Fondements}

3 Un des premiers ouvrages à avoir pour projet de faire un point sur la construction épistémologique de la didactique du français est celui co-dirigé par J.-L. Chiss, J. David et Y. Reuter (2008 [1995]) intitulé Didactique du français. Fondements d'une discipline. Son objet principal, précisé dans l'introduction, porte sur la question de l'élaboration des modèles théoriques de la didactique du français. En particulier dans son article, M. Dabène (2008 [1995], p.15-34), retrace l'histoire de l'émergence de la perspective épistémologique dans la constitution du champ de la didactique du français depuis les années 1960 à travers les colloques, les publications et la création de l'association internationale pour des recherches en didactique du français langue maternelle (AIRDFLM) créée en 1986, puis AIRDF en 2004. Les contributions reviennent sur les concepts didactiques comme par exemple celui de transposition didactique (Schneuwly, p. 47-59), celui d'interaction (Halté, 2008 [1995], p.61-75), ou encore ceux de compétences et de capacités (Bronckart, p.135-148).

4 Toutefois, dans sa synthèse conclusive, Y. Reuter (Chiss, David \& Reuter , 2008 [1995], p. 211-234), tout en réaffirmant la nécessité d'interroger le "rôle fédérateur et cristallisateur» (ibid., p.216) des concepts dans l'émergence de la didactique du français, constate le petit nombre de concepts spécifiques à la didactique et n'exclut pas qu'il puisse s'agir pour la recherche en didactique du français d'une "difficulté à conceptualiser différemment ce qui relève du langage-objet, du langage qui sert à l'enseignement-apprentissage du français, du langage qui sert à l'enseignementapprentissage dans les différentes disciplines»(ibid., p. 220). Y. Reuter revient en particulier sur le rapport entre recherche en didactique et pratiques pédagogiques. Il reprend la proposition de J.-F. Halté (2008 [1995], p. 61-75) d'interroger « la mutation de la "matrice disciplinaire" " (Chiss, David \& Reuter, 2008 [1995], p. 215) et propose de suivre A. Petitjean (2008 [1995], p. 151-167) quand il préconise de " prendre en compte [1]es pratiques dans leur histoire » (Chiss, David \& Reuter , 2008 [1995], p.216).

5 Ce questionnement sur le rapport (entre recherche en didactique et pratique pédagogique) revient à interroger, d'une part l'objet épistémique de la recherche en didactique du français : s'agit-il de la langue (en l'occurrence le français) telle qu'elle est enseignée et apprise ou de l'activité d'enseignement-apprentissage de cette 
langue? D'autre part la fonction sociale de cette recherche a-t-elle pour fonction de décrire, par le moyen d'une formalisation théorique, l'activité d'enseignementapprentissage d'une langue dans une société et un système institutionnel donnés ou de fournir des données, voire des solutions, pour "améliorer" l'enseignementapprentissage du français, d'être une didactique praxéologique selon l'expression de J.F. Halté (2008 [1995], p. 73) ? En résumé : la didactique est-elle une épistémologie située ? Une anthropologie de la transmission langagière ? Une pédagogie de la langue? Une linguistique ou une littérature appliquée ? Etc. Si l'ouvrage pose nettement et avec acuité ces questions et les enjeux correspondants, il ne fournit pas les concepts ni les modèles théoriques pour les formaliser.

\subsection{0 - Construction épistémologique vs transformation de l'école}

6 En 2001, l'ouvrage publié sous la direction de M. Marquillo Larruy réunit les contributions aux journées d'étude de la didactique du français langue maternelle (DFLM) tenues du 20 au 22 janvier 2000. Le titre est éloquent: Questions d'épistémologie en didactique du français (langue maternelle, langue seconde, langue étrangère) et indique nettement que la question de la construction épistémologique de la recherche en didactique du français revient en discussion. Cette fois la perspective est celle de la didactique des langues: en effet, le questionnement concerne aussi bien le français langue maternelle que langue étrangère ou encore langue seconde. Il est donc présupposé qu'il existe des conceptions communes entre ces différentes didactiques. Le texte de présentation de la rencontre, qui prévoit qu'un atelier du colloque (sur trois) porte sur «des questions de définitions, de modélisations et de constitution du domaine de la didactique du français: les paramètres et les facteurs à prendre en compte, les concepts utilisés et leur degré d'opérationnalité, les évolutions dans les savoirs issus des recherches produites lors de ces vingt dernières années ", met en évidence l'importance de la thématique de l'élaboration conceptuelle. Les contributeurs répondent en partie aux interrogations soulevées dans l'ouvrage précédent. Y. Reuter (p.51) réaffirme la nécessité pour une discipline «de pratiquer sérieusement une réflexion sur l'activité même de recherche si elle entretient une quelconque ambition scientifique ». Mais domine par ailleurs l'idée que, pour établir leur assise institutionnelle, les recherches en didactique du français doivent viser la transformation de l'école, même au prix d'en affaiblir la qualité scientifique (J.-F. Halté, p. $14 ;$ R. Goigoux, p. 125). Quant aux objets de recherche à privilégier pour atteindre cet objectif : certains prônent le savoir à transmettre (J.-F. Halté), d'autres l'apprenant et son activité (J.-C. Chevalier, J.-C. Beacco), d'autres encore le travail de l'enseignant et les outils qu'il mobilise (R. Goigoux). Les positions sont alors plutôt tranchées, et si le projet est commun, le moyen épistémologique de le construire ne fait pas complètement consensus.

7 C'est justement ce point que reprend C. Germain (2001, p. 295) dans son intervention finale au titre de grand témoin de la rencontre. Pour lui « dans l'atelier 1, beaucoup de propos intéressants en soi ont été émis mais, faute de s'entendre de prime abord sur les questions à examiner, il semble qu'on ait peu approfondi les questions épistémologiques posées initialement. Autrement dit, il y a eu peu d'étude systématique de questions de nature proprement épistémologique ». Selon lui l'absence de réel débat épistémologique, en particulier sur l'objet de la didactique, sur les notions 
qu'elle manipule, comme par exemple le triangle didactique, est le fait de «la visée prescriptive actuelle de la plupart des recherches en didactique qui empêche son développement scientifique " (ibid., p. 299). C. Germain conclut qu'il reste un long chemin à parcourir "pour en arriver à la constitution d'une véritable didactique du français, en supposant que l'on puisse arriver à s'entendre sur la nature de la discipline ou, à tout le moins que l'on explicite ses présupposés fondamentaux » (ibid., p. 300).

Il ressort de cet ouvrage, à la différence du précédent, que les débats épistémologiques sont fondés sur des conceptions très différentes, voire contradictoires, de l'objet de la didactique du français, et en particulier de ce qui est entendu par langue française, de ses enjeux et des modalités de son développement.

\subsection{7 - Institutionnalisation d'un corpus conceptuel}

9 La parution du Dictionnaire des concepts fondamentaux des didactiques (Reuter, 2013 [2007]) peut être considérée comme une étape marquante de l'élaboration épistémologique de la recherche en didactique dans la mesure où cet ouvrage constitue une institutionnalisation des concepts construits et mobilisés par des chercheurs en didactique. L'introduction (rédigée par Y. Reuter ; ibid., p. 5-7) précise que l'enjeu de la présentation de ce corpus conceptuel consiste à mettre en évidence la spécificité des didactiques par rapport aux autres champs de recherche s'intéressant aux questions d'enseignement, d'apprentissage et d'éducation. Il n'est pas anodin de remarquer le virage pris par rapport à la rencontre de 2000 : il ne s'agit plus de construire une épistémologie commune à la didactique des « différents français " (langue maternelle, étrangère, seconde, etc.) mais avec les « autres» didactiques des disciplines scolaires. Le lectorat potentiel mentionné est important : les chercheurs, les étudiants, les enseignants, les formateurs d'enseignants. Il s'agit donc bien d'un processus d'institutionnalisation à la fois dans le champ scientifique de la recherche en didactique et au sein du système scolaire.

Ce dictionnaire n'est pas dédié à la seule didactique du français. Parmi les quarante concepts répertoriés, six seulement peuvent être considérés comme spécifiques de la didactique du français : genres du discours, métalangage/activité métalinguistique et pratiques langagières, communauté discursive, forme scolaire et niveaux de formulation. Les trente-quatre autres concepts présentés appartiennent aux différentes didactiques des disciplines scolaires. Toutefois une certaine ambiguïté réside dans la nature conceptuelle des cinq termes suivants : didactiques, didactique comparée, didactiques et disciplines contributoires, méthodes de recherche et pédagogie, qui désignent selon nous davantage des disciplines de recherche ou des questions afférentes que des concepts didactiques. L'article « concept» fournit des explications. Les auteurs y insistent sur la distinction entre concepts scientifiques, concepts scolaires et concepts quotidiens et précisent que « si la préoccupation première des didacticiens est celle de l'étude des concepts scolaires, ainsi que celle de leur construction par l'enseignement et dans les apprentissages, elle requiert néanmoins l'étude des autres types de concepts "(ibid., p.35). Ainsi, dans ce dictionnaire des concepts des didactiques, le concept de concept renvoie davantage aux savoirs ou aux contenus scolaires pensés dans le processus de transposition didactique qu'aux concepts construisant l'épistémologie de la didactique en tant que discipline de recherche. Cette définition et cet article entrent en résonnance avec les définitions proposées à l'article 
" didactiques » qui précise que " les didacticiens sont des spécialistes de disciplines scolaires ", mais ils entrent en contradiction avec la définition suivante formulée dans le même article (ibid., p. 69)

Les didactiques sont des disciplines de recherche, c'est-à-dire des domaines scientifiques, caractérisés par des questions spécifiques (en l'occurrence la constitution, la description, les variations des contenus et de leur mise en œuvre via l'enseignement, ainsi que leurs modalités d'appropriation...), des théories, des concepts, des méthodes de recherche et des recherches empiriques (recueillant et traitant des données).

Dans ces deux définitions, le terme " concept " désigne, d'une part les contenus ou objets d'enseignement-apprentissage au sein de l'école, et d'autre part, les concepts qui structurent l'épistémologie du champ de la didactique. Finalement l'ambiguïté ou le flou noté par C. Germain en 2000 perdure, ou pire s'institutionnalise.

\subsection{8 - Le social : un enjeu ou un fondement théorique ?}

En 2008, dans La Lettre $\mathrm{n}^{\circ} 42$ (Dumortier, 2008), l'AIRDF rend hommage à J.-F. Halté, dont le rôle dans la création de l'association et dans l'élaboration épistémologique de la didactique du français a été majeur. Les contributeurs se saisissent de cette occasion pour revenir sur l'histoire (somme toute récente) du développement de la didactique du français et dresser quelques perspectives fortes. Le fil rouge de ce numéro n'est donc pas la construction épistémologique de la discipline ; toutefois trois articles abordent ce point en adoptant des points de vue différents, montrant ainsi que le débat est permanent.

Le premier point de vue est celui de J.-P. Laurent (2008, p. 7) qui insiste sur l'avancée de la construction théorique de la discipline mais prône la vigilance en ce qui concerne sa visée transformatrice.

On peut estimer qu'aujourd'hui la constitution de la didactique comme discipline scientifique disposant de ses propres théories de référence est renforcée et recentrée. On peut reconnaitre aussi que des préoccupations épistémologiques ont grandi au sein de la communauté didactique qui a conscience des limites de ses recherches et de ses champs d'action. Mais la voie ouverte à la didactique par JeanFrançois Halté, celle d'une transformation de l'enseignement-apprentissage du français dans la visée d'une société plus juste garde toute son actualité.

L'auteur, en se référant au projet déclaré lors du deuxième colloque international de didactique et de pédagogie du français tenu à Sèvres en 1983 et au cours duquel A. Petitjean et H. Romian «notaient l'urgence "d'articuler rigoureusement les forces d'innovation, les dispositifs de formation et les dispositifs de recherche" [et] appelaient de leurs vœux une didactique "construisant son identité, travaillant aux changements sociaux" » (Dumortier, 2008, p. 6), pose ainsi la question de la transformation sociale comme un fondement de la didactique du français.

Le deuxième point de vue est celui de Y. Reuter qui revient sur l'élaboration du Dictionnaire des concepts fondamentaux des didactiques, pour distinguer les trois domaines dont relèvent les concepts mobilisés en didactique du français: domaine des didactiques, domaine des disciplines dont sont issus les objets d'enseignement-apprentissage, domaine des disciplines traitant des modalités de l'enseignement et de l'apprentissage. Selon Y. Reuter (2008, p.19), cette "tripartition" abonde l'idée que soit la/les didactiques sont "des disciplines d'interaction ", c'est-à-dire qui mettent en relation des domaines et des disciplines 
selon leurs prismes propres, soit que nombre de travaux se réclamant des didactiques ont " un statut épistémologique relativement incertain, dominé, ou peu conscientisé », rejoignant en cela la conclusion de C. Germain lors des journées d'étude de 2000 (voir partie 1.2). L'auteur note également d'une part le petit nombre de concepts mobilisés, sauf trois principaux : transposition didactique, contrat didactique et triangle didactique ; d'autre part l'émergence d'une élaboration conceptuelle plus spécifique et problématisée autour des concepts par exemple de conscience disciplinaire, de modèle didactique, ou encore d'erreur (ibid., p. 20). Cette mise au point est particulièrement éclairante dans la mesure où elle débouche sur une interrogation sur le statut épistémique de/ des didactiques.

Le troisième point de vue est celui de M. Dabène et M. Rispail qui présentent une autre perspective en insistant sur la dimension fondamentalement psycho-sociale de la didactique des langues, dont celle $d u$ français Ils fondent leur réflexion sur deux concepts : celui de situation formelle et informelle d'enseignement-apprentissage des langues et celui des pratiques langagières et des représentations sociales de l'oral et de l'écrit (Dumortier, 2008, p. 10). Selon les auteurs, ces fondements épistémologiques requièrent d'inscrire les recherches en didactique du français, langue de l'école, dans « l'environnement langagier de l'enseignement-apprentissage, pratiques et représentations, intra et extra muros " (ibid., p. 11). Ce principe épistémologique conduit à concevoir "le savoir enseigné [comme] un domaine de recherche, un lieu de représentation et une pratique quotidienne de l'enseignant comme de l'élève, dans la classe et en dehors de la classe » et à concevoir l'enseignant et l'élève comme participants " en tant que sujets socialement situés à cet univers langagier, observé et vécu » (ibid., p. 11). M. Dabène et $M$. Rispail considèrent que ce fondement épsitémologique, " renoue, d'une certaine façon, avec les options idéologiques des débuts de la DFLM» (ibid., p. 12).

Ainsi dans ce numéro, sont définis trois positionnements majeurs qui structurent le champ épistémique de la didactique du français: (1) la construction d'un corpus conceptuel est nécessaire pour fonder la visée sociale transformatrice de la didactique du français; (2) le corpus conceptuel de la didactique du français est un sous-ensemble de celui plus vaste des didactiques; (3) le corpus conceptuel de la didactique du français intégré à celui de la didactique des langues est fondamentalement par nature social ou psychosocial. Le deuxième positionnement, décrit par Y. Reuter, tend à inscrire la didactique du français dans le champ épistémique des didactiques des disciplines scolaires (ibid., p. 18); alors que le troisième positionnement, proposé par $\mathrm{M}$. Dabène et $\mathrm{M}$. Rispail, tend à l'inscrire dans le champ épistémique de la didactique des langues. Cette distinction - voire ce clivage - est fondamentale et est encore aujourd'hui structurante. La question sociale est également en débat : soit elle est un enjeu (premier positionnement), soit elle est un fondement conceptuel (troisième positionnement).

\subsection{1 - 2012 - Avancées et freins}

Dans l'introduction à l'ouvrage qu'ils coordonnent en 2011 et intitulé Les Concepts et les méthodes en didactique du français, B. Daunay, Y. Reuter et B. Schneuwly s'attèlent à la question de ce qu'il faut entendre par concept et, prenant appui sur J.-C. Passeron (2006 [1990]), proposent de définir les concepts comme « des syntagmes fonctionnant comme des moyens théoriquement construits au sein de notre discipline, raisonnablement stables et opératoires, pour une appréhension du réel qu'elle vise à décrire, à expliquer ou à transformer " (Daunay, Reuter \& Schneuwly, 2011, p. 16). Ils 
précisent que «les concepts en didactique du français sont donc pour [eux] ces objets théoriques qui fondent le discours didactique et permettent une approche spécifique de faits que seul ce discours peut identifier, voire construire " (ibid., p. 16) et que «s'[ils] naissent de problèmes issus d'une interrogation d'un réel, ils ont pour fonction, en retour, de permettre de mieux problématiser ce dernier » (ibid., p. 17). Considérant que les méthodes de recherche sont étroitement liées à un cadre théorique, les auteurs insistent sur la nécessité d'« interroger ensemble concepts et méthodes » (ibid., p. 18). Ils soulèvent également la question de la naturalisation de certains concepts, par exemple celui de situation ou de travail appliqué à l'activité de l'enseignant, qu'ils corrèlent à celle de la nécessité de « dénaturaliser » certaines notions pour en interroger la valeur épistémologique, comme celles de classe, d'élève, etc. Ces questions relèvent indéniablement d'une réflexion épistémologique de fond et qui constitue une réelle avancée, dans l'esprit des propos de C. Germain dix ans plus tôt.

Ce texte aborde également deux débats corolaires et récurrents au sein de la didactique du français, dans la perspective, également récurrente, d'en construire la spécificité. Le premier débat revient à ramener la question de la spécificité de la didactique du français à la discussion sur sa finalité envisagée, soit comme la transformation de l'école et des pratiques enseignantes, soit comme une construction de connaissances sur l'action éducative "libérée de toute contingence d'action ou de pratique " (ibid., p. 22). La présentation de cette question comme une tension affranchit de toute réponse ou prise de position nette. Le second débat aboutit à établir la spécificité de la didactique du français par comparatisme en s'intéressant par exemple aux emprunts à d'autres corpus conceptuels ou aux concepts dits «nomades» (comme celui de travail) pour observer les reconfigurations épistémologiques. Les auteurs notent que, même si la didactique du français emprunte plus qu'elle ne prête (ibid., p. 25), elle parvient à construire « une armature conceptuelle » solide.

Si cet ouvrage constitue une avancée certaine et significative dans l'élaboration du corpus conceptuel de la didactique du français, il exprime aussi la difficulté pour la didactique du français à sortir des habituels débats sur sa finalité, en lien avec son ancrage institutionnel scolaire, dans une perspective transformationnelle et sur ses articulations avec les autres didactiques disciplinaires. Le numéro de Repères qui parait l'année suivante (Daunay \& Grossmann, 2012a) et qui a pour objet, comme son titre l'indique explicitement, de revenir sur vingt ans (1990-2010) de recherches en didactique du français dans les revues met en évidence sa lente et progressive autonomisation par rapport à ses ancrages institutionnels (école, institut de formation des enseignants par exemple) et aux disciplines de référence (Daunay \& Grossmann, 2012b, p. 8-9).

\subsection{Conclusion}

Ce rapide et non exhaustif tour d'horizon des textes qui ont abordé la question de la construction épistémologique de la didactique du français montre qu'elle est l'objet de débats récurrents, quelquefois "tendus ", sur des points de discussion majeurs, voire des conceptions à la limite du contradictoire. Parmi les questions récurrentes, nous pouvons retenir : la didactique du français est-elle une composante de la didactique des langues ou en est-elle distincte ? La didactique du français est-elle une composante des didactiques des disciplines scolaires ou en est-elle distincte? À quel français la 
didactique du français s'intéresse-t-elle : la langue maternelle, la langue première, la langue seconde, la langue de l'école, etc. ? La didactique du français peut-elle être à la fois une discipline scientifique et interventionniste? Faut-il s'en réjouir ou le regretter? Ces questions étaient déjà soulevées par J.-L. Chiss (1989, p. 44-52) dans le $\mathrm{n}^{\circ} 82$ de Langue française ainsi que par M. Dabène (2013, p. 137-148) dans le $\mathrm{n}^{\circ} 15$ de Recherches en didactiques. S'en réjouir parce que cette permanence des questions, voire des tensions et des conflits conceptuels, montrerait que la didactique du français ne renonce pas à interroger ses fondements ; le regretter parce que cette répétition, voire ce psittacisme, signifierait que la didactique ne parvient pas (encore) à résoudre les obstacles épistémologiques qui ont, en partie, présidé à son émergence. Le dernier congrès de l'AIRDF qui, en août 2019, a mis à son programme ce thème de la construction du corpus conceptuel de la didactique du français, montre la volonté de l'association et de la communauté des didacticiens du français à poursuivre les débats épistémologiques et engage donc plutôt à se réjouir.

\section{Comment la didactique du français appréhende-t- elle le concept de concept?}

La brève revue diachronique de la première partie tend à mettre en évidence que si la nécessité de la construction épistémologique de la didactique du français fait consensus, celle-ci est encore émergente. Ainsi le concept lui-même de concept, a-t-il jusque-là été l'objet de peu de débats ; or le concept de concept a fait l'objet de nombreuses études (voir la synthèse de Dumez, 2011) qui peuvent aider à établir quelques distinctions heuristiques. Dans cette deuxième partie, nous souhaitons observer quelques cas dans lesquels ont été explorés le concept de concept et quelques distinctions corolaires entre concept et notion, entre concept du quotidien et concept scientifique, non pas comme des objets en soi, mais pour essayer d'identifier les définitions et les réflexions qui peuvent être heuristiques et/ou opératoires pour l'élaboration du corpus conceptuel de la didactique du français.

\subsection{Quelle définition de concept les didacticiens du français retiennent-ils?}

Même si la question de l'élaboration épistémologique de la didactique du français est posée de manière récurrente, il apparait que l'adoption d'une définition commune du concept de concept n'a pas été explicitement discutée. Deux articles abordent cette question. Le premier est l'entrée «Concepts - Champs conceptuels » du Dictionnaire des concepts fondamentaux des didactiques (Reuter, 2013 [2007], p. 35-39) qui propose de considérer qu'« un concept est une construction rendant compte de caractéristiques communes à un ensemble d'objets, de faits ou de phénomènes " et qu'en tant qu' 'élément important dans les processus de construction des connaissances [...] il intéresse les didacticiens. » (ibid., p. 35). La suite de l'article s'intéresse aux concepts étudiés en classe ou autrement dit «les concepts scolaires" (Vergnaud, 1990), et pas spécifiquement aux concepts construits par et pour la recherche en didactique, et plus particulièrement celle du français ou des langues. On comprend donc ici que concept est entendu au sens d'objet d'étude. Le second article est l'introduction (voir partie 1.5) de l'ouvrage dirigé par B. Daunay, Y. Reuter et B. Schneuwly (2011). Cette fois, le 
concept de concept y est envisagé dans le cadre de la construction épistémologique de la didactique du français et est défini comme un syntagme fonctionnant comme un objet théorique construit pour appréhender le réel que vise à décrire, expliquer et transformer la didactique du français en tant que discipline de recherche (Reuter, 2013 [2007], p. 17-18). Cette définition constitue donc pour l'instant celle faisant référence pour la didactique du français. Mais si pour l'instant s'entendre sur une définition commune du concept de concept n'a pas fait l'objet d'un débat épistémologique au sein de la communauté des didacticiens du français, celle-ci en discutant de la pertinence de certains concepts a interrogé les distinctions entre concept et notion, entre concept scientifique et concept quotidien, entre concept et représentation mentale. Cette manière d'aborder le concept de concept en discutant de cas précis et contextualisés constitue une autre manière d'interroger la dimension opératoire de ces notions et concepts pour développer la recherche en didactique du français.

\subsection{Concept vs notion}

La distinction entre notion et concept est posée explicitement par C. Barré-De Miniac (2002) à propos des notions/concepts de littéracie et de rapport à l'écriture, dans le $\mathrm{n}^{\circ} 30$ de La Lettre de l'AIRDF, où elle interroge : «La littéracie est-ce une notion ou un concept?». L'auteure distingue la notion qui est " une idée utile pour appréhender le sens d'un certain nombre de constats, ou pour donner du sens à des réalités observables » (ibid., p. 27) du concept qui « dans une acception stricte, [...] implique un objet aux contours étroitement circonscrits, construit, "déconstruit" et utilisé comme élément de construction d'un ensemble théorique ». C. Barré-De Miniac en déduit que la littéracie étant multidimensionnelle et donc implicitement non circonscrite, doit être considérée comme une notion. L'auteure y voit un avantage : «Poser la littéracie comme notion donne une certaine liberté au chercheur d'explorer des pistes diverses » (ibid.) et de dépasser les "cloisonnements disciplinaires» (ibid., p. 30), dans la perspective d'articuler les recherches en psychologie, en sociologie, et en didactique. Toutefois, dans ce même numéro et en réponse à $\mathrm{C}$. Barré-De Miniac, M. Dabène met en garde sur le recours en recherche à des notions "floues", au motif que leur ancrage social et culturel, et donc leurs valeurs, en particulier pour celles relevant des questions langagières, deviendraient également flous (ibid., p. 34). Cette imprécision peut être à l'origine d'une instrumentalisation ou d'une fonctionnalisation dans les sphères institutionnelle, socioéconomique, voire socioéducative, et ainsi dévoyée (ibid.). M. Dabène (2002) constate que la notion de littéracie tend à remplacer le concept d'acculturation; or ces deux notions ou concepts ne rendant pas compte des mêmes problématiques concernant la maitrise des pratiques scripturales, il craint que le changement terminologique occulte le débat scientifique (p.36). Ici apparait nettement l'enjeu de la discussion sur la distinction notion/concept, à savoir les implications scientifiques et culturelles des choix épistémologiques.

\subsection{Concepts scientifiques vs concepts quotidiens}

Dans son ouvrage, Pensée et langage qu'il consacre au développement intellectuel de l'enfant, selon une perspective socio-historique, L. Vygotski (1985 [1934]) distingue concepts quotidiens et concepts scientifiques. Bien que construite dans le cadre spécifique de la psychologie enfantine, cette distinction, comme le suggère J.-Y. Rochex 
(1997, p. 126), peut être également intéressante «sur le plan épistémologique en ce qu'[elle rend] compte de deux modes différents de pensée ». Pour L. Vygotski, les concepts quotidiens se forment chez l'enfant dans le cadre de son activité pratique et de sa communication avec son entourage, alors que les concepts scientifiques sont assimilés dans le cadre de son activité scolaire. Ces deux systèmes se nourrissent l'un l'autre. Toutefois J.-Y. Rochex (ibid.) remarque que si «les concepts quotidiens sont riches de contenu empirique et liés à l'expérience personnelle, [...] il leur manque la visée et les instruments réflexifs qui en permettraient la prise de conscience et la formalisation» alors que les concepts scientifiques «rendent possibles prise de conscience, généralisation de second degré, réflexivité et transférabilité ", sans permettre de «se confronter avec bonheur à la singularité des situations et des expériences». À cet égard, le concept de concept quotidien est proche de celui de représentation sociale que réactive $S$. Moscovici à partir des thèses d'É. Durkheim en insistant sur leur dimension épistémologique. Selon lui les représentations sociales sont " des systèmes cognitifs qui ont une logique et un langage particuliers, des théories, des sciences sui generis, destinées à la découverte du réel et à son ordination " (Moscovici, 1969). Pour D. Jodelet (1984, p. 366), une représentation sociale est « la connaissance de sens commun, ou encore la pensée naturelle, par opposition à la pensée scientifique ». Construite "à partir de nos expériences, mais aussi des informations, savoirs, modèles de pensée que nous recevons et transmettons par la tradition, l'éducation, la communication sociale ", elle est " une connaissance socialement élaborée et partagée.» (ibid., p. 366).

Pour une discipline comme la didactique du français, qui vise à décrire et éclairer scientifiquement les situations quotidiennes d'enseignement-apprentissage du français, la distinction vygotskienne ne peut être qu'opératoire, dans la mesure où elle permet de formaliser le passage de la représentation sociale ou de l'analyse empirique des situations d'enseignement-apprentissage à laquelle procède tout enseignant (chercheur ou non chercheur) à l'analyse " scientifique » (au sens de L. Vygotski) construite par le chercheur (enseignant ou non enseignant). C'est ce que confirme par exemple l'étude de l'élaboration du concept de progression par T. Thévenaz et al. (2011), d'une part à partir de l'analyse empirique de différents plans de formation envisagés comme des (ré)organisateurs des savoirs à enseigner et à apprendre dans une triple logique épistémique, institutionnelle et chronologique, et d'autre part à partir de deux études de cas portant sur l'enseignement de la lecture. Les auteurs montrent que la progression est une composante opératoire du travail pratique de l'enseignant, avec laquelle il organise (planification, programmation, etc.) son activité et celle des apprenants et que l'appréhension du concept de progression est fortement liée à cette pratique. Ainsi la progression est-elle tout autant concept quotidien à partir duquel l'enseignant appréhende l'organisation épistémique et chronologique de son enseignement et de l'apprentissage que concept scientifique à partir duquel il problématise cette organisation. Les auteurs montrent l'intérêt et la pertinence de ce concept qui, à l'articulation de la conception pratique de l'enseignement et de la conception épistémique des objets à enseigner permet de «comprendre les principes ou lois selon lesquels sont organisés réellement les contenus dans les disciplines scolaires » (p. 90). Une même pertinence épistémologique peut être affirmée pour d'autres concepts comme par exemple celui de posture (Penloup, Chabanois \& Joannides, 2011) ou de travail enseignant (Wirthner, 2011). 


\section{Du concept à la conceptualisation} généralisations successives conduit à un "résultat paradoxal ", opéré "à coups de négations" et qui, consistant à «écarter telle ou telle détermination qui a d'abord retenu notre attention et à la soustraire au champ de la réflexion, en la taxant d'insignifiante " (ibid., p. 30), aboutit «au lieu de parvenir à une appréhension approfondie de son statut et de sa structure, [...] à n'atteindre qu'un schéma superficiel d'où seraient exclus tous les traits qui font l'originalité du cas particulier. " (ibid.). S'inspirant des méthodes d'abstraction des mathématiciens, E. Cassirer propose de ne pas négliger «tous les indices particuliers" afin de pouvoir être en capacité de "dénombrer les variations dont ils sont susceptibles" (ibid., p. 31). Ainsi, pour l'épistémologue, "le concept se caractérise, non par la "généralité" d'une image représentative, mais par la validité générale d'un principe sériel » (ibid., p. 32). Cette méthode proposée par E. Cassirer nous semble pertinente pour la didactique du français, dans la mesure où elle permet que les particularités des situations d'enseignement-apprentissage ne soient pas gommées, mais au contraire rapportées à un modèle explicatif. Elle assure l'articulation entre la dimension pragmatique et axiologique de la didactique du français et sa dimension scientifique et formalisatrice. Elle est de plus cohérente avec la dimension sociale de la didactique du français selon laquelle l'activité humaine d'enseigner et apprendre oblige à ne pas verser dans des descriptions réductrices, mais à prendre en considération les variations disciplinaires, socioculturelles, contextuelles, etc. qui structurent ce champ. La didactique du français a, pour l'instant, engagé peu de recherches pouvant relever de cette conception de E. Cassirer, sauf à notre avis la recherche Lire - écrire réalisée par l'Ifé sous la direction de R. Goigoux (2015). L'importante base de données constituée à partir d'une vaste enquête menée entre 2013 et 2015 dans 131 classes réparties sur 16 académies a permis l'observation des pratiques effectives d'un important échantillon d'enseignants et partant la prise en compte de la variété des approches singulières et des variations qu'elles expriment.

29 Toutefois, l'identification des cas particuliers et leur analyse comme des variations ne constituent que la première étape de la formalisation théorique, dont la seconde étape consiste, selon une approche synoptique, à connecter ces ensembles afin d'élaborer des modèles didactiques et des constellations conceptuelles. Ce type de démarche est par exemple mise en œuvre dans l'approche de J. Dolz \& B. Schneuwly (1998) concernant 
l'enseignement de l'oral qui met en système dans une perspective praxéologique les concepts de capacité langagière, genre formel, genre scolaire, séquence didactique ; ou encore dans celle de la socio-didactique (Dabène \& Rispail, 2008) qui met en relation les concepts de représentations, de situations linguistiques complexes, de pratiques langagières scolaires/extrascolaires, de normes/variations et de rapport à. Plus largement encore, M. Dabène (ibid., p. 27) a proposé l'élaboration d'un continuum d'enseignement - apprentissage «qui assure l'unité constitutive du champ et le relie éventuellement à des champs voisins, et des axes de variation spécifiques qui rendent compte de sa diversité ». M. Dabène identifie d'une part « des éléments organisateurs du continuum " à savoir «les descriptions de la langue et des fonctionnements des textes et des discours oraux et écrits » et «les activités cognitives à l'œuvre dans les apprentissages-acquisitions langagiers »; d'autre part deux types $d^{\prime}$ '« axes de variation » : l'axe des situations d'enseignement et l'axe des situations d'apprentissageacquisition. Un des enjeux de cette synoptisation est de favoriser une structuration du champ selon des ensembles conceptuels plutôt que selon les disciplines scolaires ou disciplines de référence. Les exemples présentés ci-dessus en montrent l'heuristique.

\subsection{Conceptualiser c'est problématiser}

La conception d'E. Cassirer engage à orienter les méthodologies de recherche en didactique $d u$ français vers les démarches empiriques et qualitatives. B. Daunay, Y. Reuter \& B. Schneuwly (2011, p. 17) insistent sur cette interaction fondatrice entre concept et méthode en lien avec la mise en problème du réel, dans la mesure où «la problématisation engage en effet des modalités d'interrogation du réel armée de moyens propres aux catégories de problèmes que le concept permet d'appréhender ». Toutefois É. Balibar (2020, p. 30-31) remarque que la "problématisation», entendue comme « la transformation d'une situation (ou d'une urgence) perçue par ses acteurs et ses porteurs en une question sans réponse immédiate, et même en fait sans formulation préétablie, par un travail critique sur l'histoire et la sémantique des discours qui la représentent en son propre sein » est en train de disparaitre de l'horizon intellectuel. Il l'impute à «la tendance [...] (favorisée par l'industrialisation et même la commercialisation de la recherche scientifique) à la réduction du concept à l'une de ses formes les plus pauvres, censée remplacer le conflit théorique par l'accumulation des vérifications statistiques au service d'objectifs institutionnellement définis, c'est-à-dire la construction de modèles "simulant" les évolutions à plus ou moins long terme des variables sélectionnées » (ibid.). Pour pallier cet appauvrissement intellectuel, É. Balibar (ibid., p. 29-30) propose l'élaboration d'une «science de problèmes »vs une « science d'objets » et qui consiste à :

décrire et interpréter la façon dont, dans le domaine des sciences humaines au moins, la remontée vers les problèmes fondamentaux (choix des catégories, délimitation de l'objet, définition des modèles de démonstration) engendre inévitablement des divergences de plus en plus radicales, qui touchent à la fois à la théorie et à la pratique [...], et obligent par conséquent à prendre parti non pas au niveau des applications ou des choix de données, mais des concepts eux-mêmes, ou mieux de l'orientation dans le conflit social qui est inhérente aux concepts.

31 Ainsi, si l'on suit É. Balibar, le concept et l'opération intellectuelle qui conduit à son élaboration ne sont pas la manifestation d'un consensus mais celle de la conflictualité par laquelle procède la connaissance (ibid., p. 33) : 
Le concept, en tant que tel, est à la fois, contradictoirement, l'unité caractéristique d'une entreprise de connaissance qui se donne pour objectif de produire l'intelligibilité des situations dont nous sommes les agents ou les patients, et le point des conflits de définition et d'interprétation inhérents à la pratique de la connaissance.

L'analyse critique menée par V. Boiron et B. Kervyn (2012) à propos des études en didactique $\mathrm{du}$ français concernant l'école maternelle illustre magistralement l'heuristique de la conflictualité décrite par É. Balibar. À partir d'une rétrospective d'articles concernant la maternelle, les deux auteures montrent que la centration des investigations des didacticiens du français sur les apprentissages en rapport avec la discipline français en négligeant les apports de la psychologie et de la pédagogie conduit à questionner la conceptualisation de l'intégration par la didactique du français. Elles proposent, d'une part de sortir d'une «vision limitative de la didactique centrée sur le sujet épistémique, et invite[nt] à étudier le sujet didactique en interaction avec l'enfant, son milieu, son "déjà-là", son développement» (ibid., p. 144), et d'autre part de "repenser le sujet didactique en maternelle, ce qui reviendrait à peut-être élargir la notion et à en tout cas y intégrer l'étude des "entours" de ce sujet (Daunay, 2011, p. 62): ses multiples dimensions (cognitive, sociale, psychologique, affective, motrice, ...) et les milieux scolaire et extrascolaire où il évolue » (ibid., p. 145). On voit, à travers cette étude, comment la mise en évidence d'un « conflit » conduit à questionner les concepts (sujet épistémique vs sujet didactique) et les orientations épistémiques de la didactique du français.

\subsection{Conceptualiser c'est construire un discours}

33 L'activité conceptualisante procédant fondamentalement par la verbalisation, il est nécessaire de considérer que la question terminologique et discursive est une des composantes de la question épistémologique (Balibar, 2016). Toutefois la question de l'élaboration du discours conceptuel ne peut consister à réduire le concept à la désignation et à la référenciation d'un objet ou d'une classe d'objets. Il s'agit plus exactement de considérer l'expression de l'activité de conceptualisation. Ainsi plus largement, la question est celle de la construction de la langue scientifique vs langue naturelle, auto-normée vs socio-normée, ahistorique vs historique (Gaudin, 1996).

Analyser l'élaboration du discours épistémologique d'une discipline de recherche relève d'une démarche de méta-recherche qui consiste à examiner de manière théorique les constructions conceptuelles en didactique du français, les modalités de leurs discussions selon une approche comparative et historique. En raison de l'important travail de brassage théorique nécessité par de telles études, celles-ci ne sont pas pléthore en didactique du français. Nous pouvons toutefois citer celle de B. Schneuwly (2008) concernant le concept de transposition didactique déjà mentionnée (voir partie 1.1) ainsi que celle de M. Jaubert, M. Rebière \& J.-P. Bernié (2003) concernant le concept de communauté discursive. Dans cette seconde étude, les auteurs montrent comment ils ont construit ce concept en examinant la genèse de l'irruption et du développement des notions/concepts de contexte, fictionnalisation, outils, postures, genres, etc. empruntés aux théories vygotskienne et bakhtinienne dans le champ de la didactique du français et en les discutant dans le cadre d'études empiriques. Ce parcours épistémologique les conduit non seulement à interroger ces concepts au regard des problèmes posés par l'enseignement-apprentissage du français, mais également à, inversement, mettre en évidence que l'activation de ces concepts 
dans les études didactiques est en adéquation avec une conception complexe de la didactique du français et le rejet de la réduire à une ingénierie. Ces méta-recherches visant à mettre en lumière l'élaboration du discours conceptuel en didactique du français doivent permettre de mettre en évidence l'émergence et la circulation des concepts entre les différents champs scientifiques reliés à celui de la didactique du français, d'une part en identifiant d'où viennent les concepts construits dans le champ de la didactique du français par le biais d'emprunts à d'autres didactiques et/ou à d'autres champs épistémiques (en particulier des sciences humaines et sociales); d'autre part en explorant leur rayonnement dans les autres didactiques, ou dans les autres champs disciplinaires et, dans une perspective plus praxéologique, dans les domaines d'application de la didactique du français tels que l'enseignement et la formation, les référentiels, etc. À l'évidence, ces méta-recherches discursives vont devoir constituer un axe important dans le domaine de la didactique du français.

\section{Conclusion}

Bien que loin d'être exhaustive - elle ne peut pas l'être -, cette synthèse met en évidence que la construction épistémique de la didactique du français est une question vivement débattue depuis la constitution du champ, soit en tant que telle, soit à partir d'études de cas. Cette vivacité manifeste une certaine vigueur épistémologique, non par goût de la joute théorique - encore que..., mais plutôt en raison des fondements qui ont présidé à la constitution de ce champ épistémique et qui, nous semble-t-il, sont encore en débat. Ils peuvent se résumer en deux questions : (1) en raison de la nature de son objet d'étude, l'enseignement-apprentissage de la langue française, la didactique du français est-elle un sous-ensemble de la/des didactiques des disciplines scolaires ou estelle une composante de la didactique des langues? (2) la question sociale est-elle la visée de la didactique du français, et ainsi l'inscrit-elle dans le champ épistémique des sciences ingéniériques et/ou transformatrices des pratiques humaines ou en est-elle le fondement épistémique, et ainsi l'inscrit-elle dans le champ des sciences psychosociales ? Poser ainsi cette double question est sans doute simplificateur. Celle-ci reste toutefois, selon nous, aujourd'hui institutionnellement et théoriquement structurante dans le développement de la didactique du français.

\section{BIBLIOGRAPHIE}

BALIBAR, É. (2016). « Le concept de concept : "un se divise en deux" ». Colloque Political concepts: The Balibar Edition at Brown University, Providence, 2-3 déc. En ligne : https:// www.politicalconcepts.org/concept-etienne-balibar/.

BALIBAR, É. (2020). Passions du concept. Épistémologie, théologie, politique. Écrits II. Paris : Éd. La Découverte. 
BARRÉ-DE MINIAC, C. (2002). «La notion de littéracie et les principaux courants de recherche ». La Lettre de l'AIRDF 30, p. 27-33. En ligne : https://www.persee.fr/doc/

airdf_1260-3910_2002_num_30_1_1519.

BOIRON, V. \& KERVYN, B. (2012). «École maternelle et recherches en didactique du français : bilan rétrospectif ». Repères. Recherche en didactique du français langue 46, p. 131-148. En ligne : https:// doi.org/10.4000/reperes.97.

CASSIRER, E. (1977) [1910]. Substance et fonction. Éléments pour une théorie du concept. Trad. de l'allemand par P. Caussat. Paris : Éditions de Minuit.

CHISS, J.-L. (1989). « Revendication d'autonomie et horizon de scientificité en didactique du français ». Langue française, 82, p. 44-52. En ligne : https://www.persee.fr/doc/

lfr_0023-368_1989_num_82_1_6380.hM.

CHISS, J.-L., DAVID, J. \& REUTER, Y. (2008) [1995]. Didactique du français. Fondements d'une discipline. Bruxelles : De Boeck.

DABÈNE, M. (2002). « Réactions à la "notion" de littératie ». La Lettre de l'AIRDF 30, p. 33-36. En ligne : https://www.persee.fr/doc/airdf_1260-3910_2002_num_30_1_1520.

DABÈNE, M. (2008) [1995]. « Quelques repères, perspectives et propositions pour une didactique du français dans tous ses états ». In : Chiss, J.-L., David, J. \& Reuter, Y. Didactique du français. Fondements d'une discipline. Bruxelles : De Boeck, p. 15-34.

DABÈNE, M. (2013). « Questions et débats sur l'histoire de la didactique du français. 1965-2007 : quelque 40 ans de déambulations dans les territoires de la didactique du français. Entretien avec Michel Dabène ». Recherches en didactiques, 15, p. 137-148. En ligne : https://www.cairn.info/ revue-recherches-en-didactiques-2013-1-page-137.htm

DABÈNE, M. \& RISPAIL, M. (2008). « La sociodidactique : naissance et développement d'un courant au sein de la didactique du français en France ». La Lettre de l'AIRDF 42, p. 10-13. En ligne : https:// www.persee.fr/doc/airdf_1776-7784_2008_num_42_1_1769.

DAUNAY, B. (2011). « L'enfant, l'élève, l'apprenant en didactique du français ». Recherches en didactiques 11, p. 49-65. En ligne : https://doi.org/10.3917/rdid.011.0049.

DAUNAY, B., REUTER, Y. \& SCHNEUWLY, B. (2011). Les Concepts et les méthodes en didactique du français. Namur : Presses universitaires de Namur.

DAUNAY, B. \& GROSSMANN, F. (coords) (2012a). « Vingt ans de recherches en didactique du français (1990-2010) ». Repères. Recherche en didactique du français langue 46. En ligne : https://doi.org/ 10.4000/reperes.74.

DAUNAY, B. \& GROSSMANN, F. (2012b). « Vingt ans de recherches en didactique du français (1990-2010). Quelques aspects des recherches dans les revues ». Repères. Recherche en didactique du français langue 46 p. 7-16. En ligne : http://reperes.revues.org/82

DOLZ, J. \& SCHNEUWLY, B. (1998). Pour un enseignement de l'oral. Initiation aux genres formels à l'école. Issyles-Moulineaux : ESF éd.

DUMEZ, H. (2011). « Qu'est-ce qu'un concept ? ». Le Libellio d'AEGIS 7 (1), p. 67-79. En ligne : https:// hal.archives-ouvertes.fr/hal-00574166.

DUMORTIER, J.-L. (éds) (2008), La Lettre de l'AIRDF 42. En ligne : https://www.persee.fr/issue/ airdf_1776-7784_2008_num_42_1.

GAUDIN, F. (1996). « Terminologie : l'ombre du concept ». Méta 414, p. 604-621. 
GERMAIN, C. (2001). « Didactique du français et épistémologie. De la nécessité d'expliciter ses présupposés ». In : Marquillo Larruy, M. (éds). Questions d'épistémologie en didactique du français (langue maternelle, langue seconde, langue étrangère). Poitiers : Université de Poitiers, p. 295-300. GoIGouX, R. (dir.). (2015). Lire et écrire. Étude de l'influence des pratiques d'enseignement de la lecture et de l'écriture sur la qualité des premiers apprentissages. Rapport de recherche. En ligne : http:// ife.ens-lyon.fr/ife/recherche/lire-ecrire/rapport/rapport.

GUERNIER, M.-C. (2020). « Les concepts dans la recherche en didactique du français. Émergence et création d'un champ épistémique. Un regard sur les propositions de communications ». La Lettre de l'AIRDF 67.

HALTÉ, J.-F. (2008) [1995]. «Interaction : une problématique à la frontière ». In : Chiss, J.-L., David, J. \& Reuter, Y. Didactique du français. Fondements d'une discipline. Bruxelles : De Boeck, p. 61-75.

JAUBERT, M., REBIÈRE, M. \& BERNIÉ, J.-P. (2003). « L'hypothèse “ communauté discursive” : d'où vientelle ? Où va-t-elle?». Les cahiers Théodile 4, p. 51-80. En ligne : https://red-revue.univ-lille.fr/lescahiers-theodile-n4.

JODELET, S. (1984). « Représentation sociale : phénomènes, concepts et théorie ». In : Moscovici, S. (éd.). Psychologie sociale. Paris : Presses universitaires de France, p. 363-384.

LAURENT, J.-P. (2008). « L'émergence (difficile) d'une discipline d'argumentation. Un aspect de la préhistoire de la didactique du français en Belgique francophone ». La Lettre de l'AIRDF 42, p. 3-7. En ligne : https://www.persee.fr/doc/airdf_1776-7784_2008_num_42_1_1766.

MARQUILLO LARRUY, M. (dir.). (2001). Questions d'épistémologie en didactique du français (langue maternelle, langue seconde, langue étrangère). Poitiers : Université de Poitiers.

MoscovicI, S. (1969). « Préface ». In: Herzlich, C. Santé et maladie. Analyse d'une représentation sociale. Paris/La Haye : Éd. Mouton, p. 7-12.

PASSERON, J.-C. (2006) [1990]. Le Raisonnement sociologique. Paris : Nathan.

PENLOUP, M.-C., CHABANOIS, M. \& JOANNIDÈS, R. (2011). « La "posture" : effet de mode ou concept pour la didactique du français ». In : Daunay, B., Reuter, Y. \& Schneuwly, B. Les Concepts et les méthodes en didactique du français. Namur : Presses universitaires de Namur, p. 151-176.

PETITJEAN, A. (2008) [1995]. « Variations historiques : l'exemple de la rédaction ». In : Chiss, J.-L., David, J. \& Reuter, Y. Didactique du français. Fondements d'une discipline. Bruxelles : De Boeck, p. $151-168$

REUTER, Y. (2008). «Interroger l'appareillage conceptuel de la didactique du français ». La Lettre de l'AIRDF 42, p. 18-21. En ligne : https://www.persee.fr/doc/airdf_1776-7784_2008_num_42_1_1773. REUTER, Y. (ed.). (2013) [2007]. Dictionnaire des concepts fondamentaux des didactiques. Bruxelles : De Boeck.

ROCHEX, J.-Y. (1997). « L'œuvre de Vygotski : fondements pour une psychologie historicoculturelle ». Revue Française de Pédagogie 120, p. 105-147. En ligne : https://doi.org/10.3406/rfp. 1997.1161.

THÉVENAZ, T., et al. (2011). « Progression : un concept fondateur de la didactique. L'exemple de la lecture à travers l'école obligatoire ». In : Daunay, B., Reuter, Y. \& Schneuwly, B. Les Concepts et les méthodes en didactique du français. Namur : Presses universitaires de Namur, p. 85-116.

VERGNAUD, G. (1990). « La théorie des champs conceptuels ». Recherche en didactique des mathématiques, vol.10, 23, p. 133-170. 
VYGOTSKI, L.S. (1985) [1934]. Pensée et langage. Trad. du russe de F. Sève. Paris : Éditions Sociales.

WIRTHNER, M. (2011). « Travail de l'enseignant et méthodes de recherche en didactique du français ». In Daunay, B., Reuter, Y., \& Schneuwly, B. Les Concepts et les méthodes en didactique du français. Namur : Presses universitaires de Namur, p. 117-148.

\section{RÉSUMÉS}

Pour se constituer scientifiquement comme champ de recherche, la didactique du français doit engager un travail épistémologique d'élaboration de concepts. C'est la problématique mise en débat par l'AIRDF lors de son $14^{\mathrm{e}}$ congrès en août 2019 à Lyon et dont cet article propose une synthèse. La question est abordée selon trois aspects : selon l'aspect diachronique nous observons comment la question de la conceptualisation en didactique du français est examinée dans des publications fondatrices. Selon l'aspect épistémologique nous examinons d'une part comment le concept de concept y est appréhendé (aspect $\mathrm{n}^{\circ} 2$ ), et d'autre part, quelle heuristique développe l'activité de conceptualisation (aspect $\mathrm{n}^{\circ} 3$ ).

In order to establish itself scientifically as a field of research, the didactics of french language must engage an epistemological work to develop concepts. This is the issue debated by the AIRDF at its $14^{\text {th }}$ congress, in August 2019, in Lyon and which this article summarises. The issue is approached from three angles. With the diachronic aspect we observe how the question of conceptualisation in didactics of french langage is examined in founding publications. With the epistemological aspect we examine, on the one hand, how the concept of concept is apprehended there (aspect $\left.n^{\circ} 2\right)$, on the other hand, which heuristic develops the activity of conceptualisation (aspect $\mathrm{n}^{\circ} 3$ ).

\section{INDEX}

Mots-clés : didactique du français, champ épistémique, concept, conceptualisation Keywords : didactics of French, epistemic field, concept, conceptualisation

\section{AUTEUR}

\section{MARIE-CÉCILE GUERNIER}

Université Claude Bernard Lyon 1, Polytech Lyon, F-69622 Villeurbanne, FranceUniversité Grenoble Alpes, Lidilem, F-38058 Grenoble, France 